\title{
A Motivational-Developmental Free Response Assessment Through a Bifactor Lens
}

\author{
David Alpizar and Brian F. French* \\ Learning and Performance Research Center, Washington State University, Pullman, WA, United States
}

OPEN ACCESS

Edited by: Karl Schweizer,

Goethe University Frankfurt, Germany

Reviewed by:

Rudolf Debelak,

University of Zurich, Switzerland

Seung W. Choi,

University of Texas at Austin,

United States

*Correspondence:

Brian F. French

frenchb@wsu.edu

Specialty section:

This article was submitted to

Quantitative Psychology and Measurement,

a section of the journal

Frontiers in Psychology

Received: 03 September 2021 Accepted: 10 November 2021 Published: 03 December 2021

Citation:

Alpizar D and French BF (2021) A Motivational-Developmental Free Response Assessment Through a Bifactor Lens.

Front. Psychol. 12:770327. doi: 10.3389/fpsyg.2021.770327
The Motivational-Developmental Assessment (MDA) measures a university student's motivational and developmental attributes by utilizing overlapping constructs measured across four writing prompts. The MDA's format may lead to the violation of the local item independence (LII) assumption for unidimensional item response theory (IRT) scoring models, or the uncorrelated errors assumption for scoring models in classical test theory (CTT) due to the measurement of overlapping constructs within a prompt. This assumption violation is known as a testlet effect, which can be viewed as a method effect. The application of a unidimensional IRT or CTT model to score the MDA can result in imprecise parameter estimates when this effect is ignored. To control for this effect in the MDA responses, we first examined the presence of local dependence via a restricted bifactor model and Yen's Q3 statistic. Second, we applied bifactor models to account for the testlet effect in the responses, as this effect is modeled as an additional latent variable in a factor model. Results support the presence of local dependence in two of the four MDA prompts, and the use of the restricted bifactor model to account for the testlet effect in the responses. Modeling the testlet effect through the restricted bifactor model supports a scoring inference in a validation argument framework. Implications are discussed.

Keywords: local item dependence, restricted bifactor, testlet, method effect, factor analysis

\section{A MOTIVATIONAL-DEVELOPMENTAL FREE RESPONSE ASSESSMENT THROUGH A BIFACTOR LENS}

Student's motivational and developmental (MD) attributes are vital for student adjustment, learning outcomes, achievement, and retention in higher education (Farrington et al., 2012; Baars et al., 2015; Cromley et al., 2015; Kaplan and Patrick, 2016). These attributes include leadership, self-concept, self-set goals, attribution, and other skills related to student success. Because the MD attributes are connected to students' outcomes, researchers and practitioners are using assessments to study how the MD attributes enable students to succeed in university settings (García, 2016).

Traditionally, self-report questionnaires and performance tasks are used to assess the MD attributes (Fernandez-Berrocal and Extremera, 2006; Duckworth and Yeager, 2015). The accuracy of the estimated ability with these traditional formats has been questioned in recent years for two reasons (National Academies of Sciences, Engineering, and Medicine, 2017). First, self-report questionnaires depend on the participant's answer to one's own behaviors, beliefs, or attitudes (Duckworth and Yeager, 2015; Crossley, 2016). As a result, items in self-report questionnaires can 
be misinterpreted by the participants, which can lead to inaccurate scores. Second, performance tasks rely on participants' behavior under a situation that might not reflect a realistic situation (Duckworth and Yeager, 2015; Crossley, 2016). Consequently, the participants' behavior in this format might not demonstrate how the MD attributes are manifested in their personal experience. Given traditional assessment formats might fail to obtain accurate scores for the MD attributes, the Motivational-Developmental Assessment (MDA) was developed in response to this concern.

Unlike the traditional assessment formats, the MDA consists of four open-ended writing prompts (Farrington et al., 2012; Kaplan and Patrick, 2016). Each writing prompt asks students to write about their life events, based on their personal experience. Trained raters score the students' responses on the following six MD domains: self-awareness, self-authorship, coping, selfconcept, self-set goals, and attributions of success or failure. Each writing prompt assesses three out of six MD attributes, but each skill is measured once in two prompts. Thus, the MDA format allows for a deeper understanding of how the students' MD attributes are manifested in their personal experiences compared to more traditional rating scale formats. However, the format of the prompts may invoke a method effect in the form of a testlet effect.

\section{Method Effect}

A method effect is unexplained variance beyond what is intended to be measured (Podsakoff et al., 2003, 2012). The method effect results from any aspect of a measurement process (e.g., different administration methods) and the test, including having a common measurement context, item context, or features of items (Podsakoff et al., 2003). As result, there are many types of method effects, including an order effect, a testlet effect, and rater effect (Maul, 2013). Once a method effect is identified in the data, it is possible to control for these effects, and avoid inaccurate parameter estimates (Podsakoff et al., 2012).

Different statistical approaches are developed to control for a method effect (e.g., Podsakoff et al., 2003, 2012; Maul, 2013). One way to model a method effect is using the confirmatory factor analysis (CFA) framework (Podsakoff et al., 2003). Conceptually, the method effect results in extra covariation not due to the assessed construct but reflects method effect variance. That is, method effect is an undesired latent variable not assessed by the test. Using the CFA framework, models (e.g., multi-trait multi-method CFA models) were developed, where a secondary latent variable could be estimated to control for a method effect (Podsakoff et al., 2003; Maul, 2013).

\section{Testlet Effect and Local Item Independence}

A testlet effect is a type of method effect that occurs with the use of testlets (Maul, 2013). A testlet is a set of items clustered around the same stimuli (Wainer and Kiely, 1987; Rosenbaum, 1988). This effect results from violations of the local item independence (LII) assumption, a fundamental assumption related to classical test theory (CTT) and item response theory (IRT) (Yen, 1993;
Schroeders et al., 2014; Raykov et al., 2015). This assumption is a prerequisite for IRT unidimensional models (de Ayala, 2009, p. 20) and has been the focus in simulation (e.g., Bradlow et al., 1999) and applied studies for IRT (e.g., Schroeders et al., 2014; Baldonado et al., 2015). For IRT, the LII assumption states that the probability of responding to an item relies only on the person's ability to answer each question, and the item characteristics (Lord and Novick, 1968, p. 361). Thus, the probability of a response to an item does not influence the probability of responding to other items, controlling for ability. In the context of factor analysis, this assumption is known as the uncorrelated residuals assumption (Yen, 1993; Schroeders et al., 2014), where the indicator residuals are not correlated in the factor model. Overall, meeting the LII assumption indicates that item responses are solely explained by the latent variable(s) intended to be measured by the test.

Test formats might lead to violations of the LII assumption (Yen, 1993). One of these formats is the free-response format (Ferrara et al., 1997, 1999), such as with the MDA. We note that the MDA was not purposefully constructed with testlets. Responses are nested within the MDA's prompts, similar to a testlet. In this situation, residuals within the testlets might be correlated. Consequently, local item dependence (LID) is present in the data, which can result in the testlet effect. In line with a method effect, the additional covariation between responses in a testlet might not be due to the measured construct but reflects testlet variance. Specifically, the testlet effect can be conceptualized as a secondary variable not measured by a test, but rather captured due to the administration of testlets. This secondary unmeasured variable might impact the performance of the examinee but could be accounted for in CFA models.

Ignoring the testlet effect in a scoring model can result in inaccurate parameter estimates (Koziol, 2016) and overestimating reliability estimates (DeMars, 2006). A unidimensional IRT model, for example, overestimates the discrimination parameter compared to a model that accounts for the testlet effect (Bradlow et al., 1999; Glas et al., 2000; Koziol, 2016). This overestimation can bias ability estimates and lead to possible incorrect decisions about the individual (Bradlow et al., 1999; Koziol, 2016). The inaccurate estimates are not exclusively tied to IRT. If models ignore the testlet effect, estimates can be inaccurate for factor analysis (Luo and Gordon-Wolf, 2019). Also, some reliability approaches based on CTT cannot be estimated in the presence of LID, such as the testlet effect (Raykov et al., 2015). To ensure test score accuracy in practice, the detection of LID is essential, and models should account for the testlet effect. Given this, bifactor models may assist with the detection of LID and modeling spuriously correlated responses. However, to date, this modeling strategy has been rarely used for such a purpose in educational and psychological research.

\section{Bifactor Models for the Testlet Effect}

Traditionally, items are aggregated to create a single item (i.e., polytomous-item, super-item, or parcel) for each testlet (e.g., Hall et al., 1999; Bandalos and Finney, 2001). This super-item might control the effects of LID (or testlet effect) for the items within the bundle (Eckes, 2011). Next, these super-items are assessed via the unidimensional model. This model is used to 
support a scoring inference for test scores (e.g., Eckes, 2011). However, such practice ignores the items' response pattern within the testlet. Given items within a group are aggregated to a superitem, parameters for each item (e.g., loadings) are not estimated (Wainer and Wang, 2000), but instead reflected in the super-item parameter estimates. Consequently, assessing each item's quality in terms of item specific parameters might not be possible, and the estimated ability might not be accurate (e.g., low reliability, loss of information about the examinee's ability). Given the above concerns, the testlet effect variable can be modeled as a specific factor(s) in CFA models (Maul, 2013). Though other models that account for the testlet effect are available (e.g., correlating residuals within a testlet, a second-order factor model; Braeken et al., 2007; Rijmen, 2010), we focus on two types of bifactor models (BM) to model the testlet effect, the conventional BM and the restricted $\mathrm{BM}$.

Figure 1 presents the conventional BM and restricted BM. The conventional BM specifies a general factor related to all items to explain all the item responses (Gibbons and Hedeker, 1992), and a specific factor(s), which are related to certain sets of items within a testlet (DeMars, 2006; Hernandez-Camacho et al., 2017). Conceptually, the specific factors, not measured by the assessment, represents the testlet effect. Often, the factors are specified with a mean of 0 and a variance of 1 (e.g., Rijmen, 2010), and are orthogonal to each other (Gibbons and Hedeker, 1992). This specification results in two factor pattern coefficients for each item in the conventional BM, which are all freely estimated. The first pattern coefficient represents the relationship between the item and the general factor that is assessed. The second pattern coefficient represents the association between an item within a bundle and the corresponding specific factor(s) (Koziol, 2016; Hernandez-Camacho et al., 2017). The conventional BM takes the form (e.g., Muthén and Asparouhov, 2002):

$$
y_{i}^{*}=\nu+\Lambda_{y} \eta_{i}+\epsilon_{i}
$$

where $\Lambda_{y}$ represents the pattern coefficients for the general factor and specific factor(s), and the $\nu$ is the intercept parameter. The $\eta$ represents the vector for the general factor and specific factor(s). The $\epsilon$ represents a vector of the unique variance for each item that is not explained by the general and specific factor(s). The $y^{*}$ represents a continuous latent response variable for each observed categorical item i (Muthén and Asparouhov, 2002).

An alternative model to assess testlet data with LID is the restricted BM. The restricted BM, known as the testlet model, was originally developed from an IRT framework (Bradlow et al., 1999) and then connected to CFA (Luo, 2018). This model is proposed to detect LID and provide a scoring model that accounts for the testlet effect (Bradlow et al., 1999; Li et al., 2006). Like the conventional BM, all items are related to a general factor, and items within a testlet are associated with a specific factor(s), where again the factors are orthogonal.

In comparison to the conventional $\mathrm{BM}$, the restricted $\mathrm{BM}$ requires two specifications in Equation (1). First, the general factor can be specified with a mean of 0 and variance of 1 , whereas the specific factors are specified with a mean of 0 and random variance. The random variance on a specific factor provides the estimates for the random testlet effect parameter. This variance can quantify the magnitude of LID for each testlet (Bradlow et al., 1999). Second, each corresponding specific factor's loadings are held equal to its respective loading on the general factor (Luo, 2018). Given these restrictions, this model is more parsimonious than the conventional BM (Hernandez-Camacho et al., 2017).

The conventional and restricted $\mathrm{BM}$ are connected in three ways. First, both models have the potential to assess testlet data that violate the LII assumption. Second, both models explain the item's responses with a general factor and specific factor(s) for testlet data. Third, the restricted BM is nested within the conventional BM (Hernandez-Camacho et al., 2017). These models can assist with understanding the structure and scoring of the MDA and may be more promising in evaluating correlated responses than the unidimensional models with super-items (Hernandez-Camacho et al., 2017). Thus, the BM models have the potential to provide accurate estimates for the MDA scores while controlling for the testlet or method effect.

\section{Purpose}

A single score is used for the MDA in practice (Kaplan and Patrick, 2016). This assumes that the one-factor model explains the MDA's responses, and item's responses within a writing prompt are independent. Given the MDA has a free-response format assessment, the MD attributes are nested within each writing prompt like a testlet. Thus, there can be the presence of a testlet effect for such data. In such a situation, ignoring the testlet effect can lead to imprecise parameter estimates for the MDA's one factor model (e.g., Koziol, 2016). Thus, there is a need to evaluate the LII assumption with the MDA writing prompts. Given this, the BM models can be used to document the extent LID is present for each MDA writing prompt, model the one-factor MDA structure, and control the testlet effect, if indeed detected.

Given evidence is needed about the LII assumption and a scoring claim for the MDA scores, our purpose was to investigate a scoring inference for the MDA scores via BM models to detect LID and control for the testlet or method effect. A scoring inference informs how the MDA's responses are related to the general factor (Kane, 2013), and how practitioners and researchers score the MDA. Two research questions (RQ) were addressed in this study.

RQ1: Is LID present in the MDA prompts as assessed with the restricted BM and other indices? We expect that the LII assumption will be violated, as detected by the restricted BM and Yen's Q3 index.

RQ2: Are responses for the MDA prompts explained by a unidimensional model, or by a unidimensional model that controls for the testlet effect? We investigated evidence to support a scoring inference for the MDA scores via factor analyses.

Results provide insight into the degree of support for the LII assumption and scoring claim for the MDA scores. The study also demonstrates how to detect LID and model correlated residuals via the conventional $\mathrm{BM}$ and restricted $\mathrm{BM}$. The latter model is an innovative way to account for the testlet effect. 

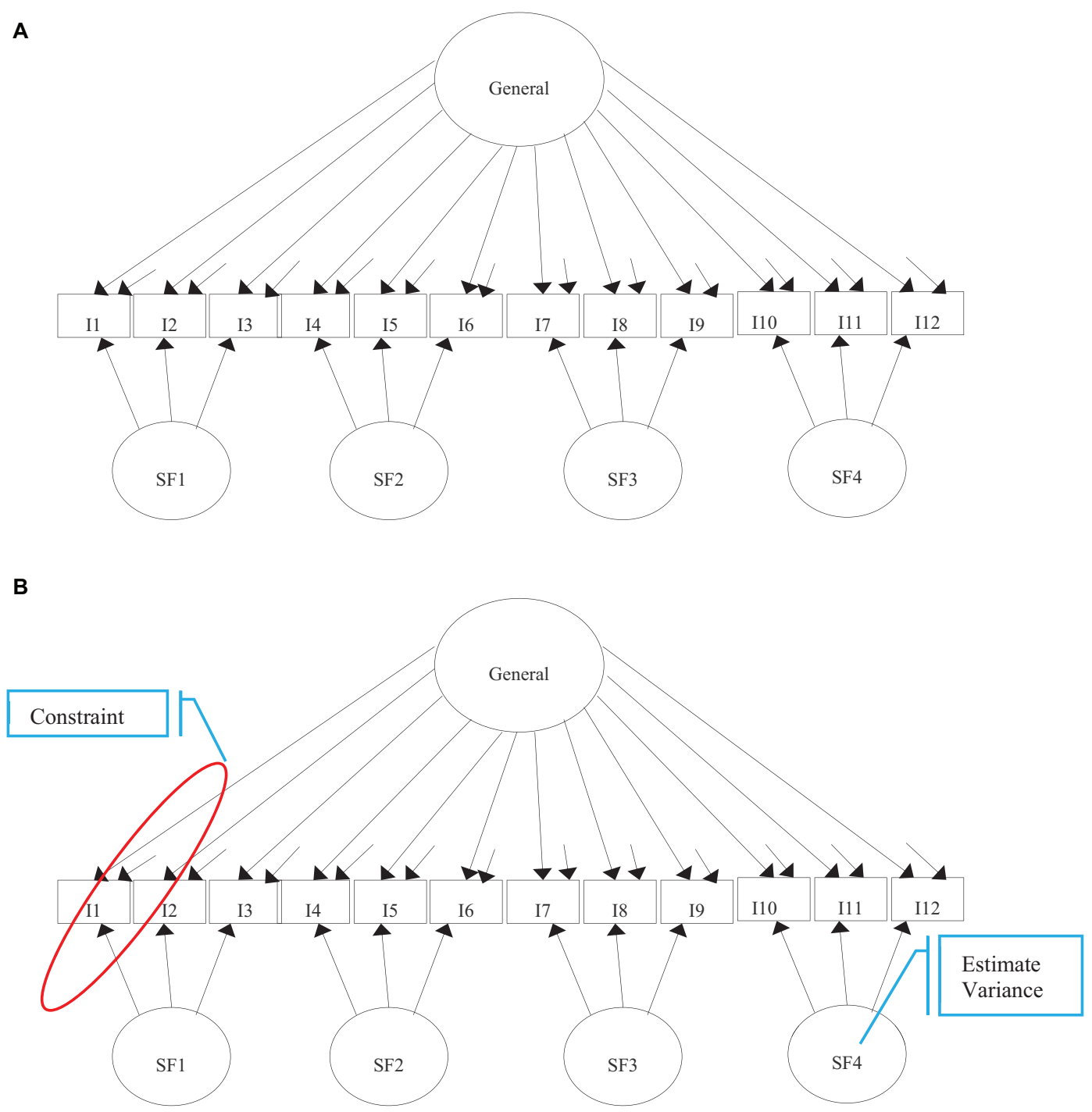

FIGURE 1 | Path diagrams of the tested models. (A) Conventional bifactor. (B) Restricted bifactor. SF, Specific Factors.

\section{MATERIALS AND METHODS}

\section{Sample and Procedure}

The sample $(N=257$; female $=75.4 ; 38.1 \%$ first generation $)$ included first-year university students from a large researchfocused university on the West coast of the United States. The assessment was completed in the first 4 weeks of the Fall 2018 semester. The assessment required approximately $10 \mathrm{~min}$ to complete.

\section{Instrument}

The MDA (Kaplan and Patrick, 2016) consists of four selfreflecting writing prompts, which ask students to write brief paragraphs about their life events. Three out of six constructs are measured with each prompt. Hence, responses are nested within each writing prompt. Trained raters require approximately $10 \mathrm{~min}$ to score all prompts for each student. Each writing prompt for the MDA assesses different MD attributes. Prompt 1 assesses self-concept, self-set goals, and attributions of success. Prompt 2 assesses self-concept, attributions of failure, and coping. Prompt 3 assesses self-awareness, self-set goals, and self-authorship. Prompt 4 assesses self-awareness, coping, and self-authorship. The correlations between the six measured attributes ranged from 0.19 between self-authorship and self-concept to 0.73 between self-authorship and self-awareness, with a median correlation of 0.34 among all attributes.

\section{Raters and Rater Calibration}

Three raters were trained and completed calibration in four sessions. First, raters were trained on the constructs and codebook by the MDA author. Second, raters practiced the ratings with examples. Third, raters met with the MDA author to discuss the inconsistencies in the practiced ratings and clarifying domain meaning. Finally, raters met consensus on 
scoring. Raters scored all responses over 10 weeks. To assess reliability of the MDA, we applied Generalizability Theory (Gtheory). This allowed us to estimate reliability for the total score, and estimate variance associated with the object of measurement (i.e., the student), and variance associated with the raters and residual variance capturing error and other possible other facets not included in the design. G-theory analysis revealed that for MDA total score: (a) reliability (Gcoefficient) was 0.91 , (b) $72.8 \%$ of the variance was associated with the student, (c) $5.9 \%$ of the variance was explained by the rater, and (d) $21.3 \%$ variance was associated with the residuals.

Reliability values met the criteria to use scores for research purposes (i.e.,>0.80; Nunnally and Bernstein, 1994), and at least two raters (i.e., $>0.80$ ) were needed to maintain adequate reliability levels. Tables $\mathbf{1}$ and $\mathbf{2}$ contain the descriptive statistics for the MDA total score and the six attributes scored across prompts, and for the individual attributes, respectively. As seen in Table 1, variability was similar across the attributes when scored across prompts. Attribution, self-authorship, and coping appeared to have the highest means out the six scores. Table 2 reveals that the attributes tended to be scored as present in prompt 1 and 2 at a higher rate compared to prompt 3 and 4 .

\section{Analysis}

For RQ1, the LII assumption was examined using the Yen's Q3 (Yen, 1984) via the subscore package (Dai et al., 2019) in the R

TABLE 1 | Descriptive statistics for the motivational and developmental (MD) total score and attribute scores across prompts.

\begin{tabular}{lccccc}
\hline MD scores & N & M & SD & Min & Max \\
\hline Total score & 257 & 5.64 & 3.26 & 0.00 & 12.00 \\
Self-concept & 257 & 0.85 & 0.73 & 0.00 & 2.00 \\
Self-set goals & 257 & 0.88 & 0.75 & 0.00 & 2.00 \\
Attribution & 257 & 1.28 & 0.78 & 0.00 & 2.00 \\
Coping & 257 & 0.99 & 0.74 & 0.00 & 2.00 \\
Self-awareness & 257 & 0.65 & 0.79 & 0.00 & 2.00 \\
Self-authorship & 257 & 1.00 & 0.87 & 0.00 & 2.00 \\
\hline
\end{tabular}

MD = Motivational and Developmental.

TABLE 2 | Percentage of students displaying an MD attribute by prompt.

\begin{tabular}{llll}
\hline Prompt & Attributes & N & Percentage \\
\hline 1 & Self-concept & 257 & 60.70 \\
1 & Self-set goals & 257 & 49.42 \\
1 & Attribution & 257 & 66.93 \\
2 & Self-concept & 257 & 24.12 \\
2 & Attribution & 257 & 60.70 \\
2 & Coping & 257 & 47.47 \\
3 & Self-Awareness & 257 & 26.07 \\
3 & Self-set goals & 257 & 38.91 \\
3 & Self-authorship & 257 & 52.14 \\
4 & Self-Awareness & 257 & 38.52 \\
4 & Coping & 257 & 51.36 \\
4 & Self-authorship & 257 & 47.47
\end{tabular}

statistical system and the restricted BM via using Mplus (Muthén and Muthén, 2017). We added the Q3 to the detection of LID because it is a common index for LID detection and provide a confirmation check with the restricted BM results. Q3 is the correlation between residuals $(d)$ for item $i$ and item $j$. Q3 is expressed as:

$$
Q_{3}=r_{d_{i} d_{j}}
$$

The detection of LID was conducted in two ways. First, following recommendations (Ferrara et al., 1997; Schroeders et al., 2014; Baldonado et al., 2015), we estimated the averages of the Q3 values and the number of pairs with LID for each prompt. LID was determined to be present if Q3 values $\geq 0.2$ (Yen, 1984) for any three pairs of items in a prompt. Second, we estimated the variance of the testlet effect using the restricted BM. This variance identified LID for each writing prompt with the following criteria (Bradlow et al., 1999): 0.1-0.5 = low LID, 0.6-1.0 = moderate LID, and 1.1-1.5 = high LID. We recognize these criteria are guidelines and lack strong empirical support (e.g., Christensen et al., 2017). We still include these, given their use in practice, with a recognition that future work is needed to support these criteria.

For RQ2, a one-factor model, a conventional BM, and a restricted $\mathrm{BM}$ were examined for the MDA using Mplus (Muthén and Muthén, 2017), with the robust weighted least square estimator (WLSMV) to account for dichotomous data. Sample codes for estimating each model are provided in the supplementary material. The one-factor model ignored the testlet effect and served as a baseline to compare the BM models' results. The conventional $\mathrm{BM}$ with four specific factors and the restricted $\mathrm{BM}$ with four specific factors were tested, following the specifications described in the introduction. These models were selected because they were alternative models to account for the testlet effect (e.g., DeMars, 2006; Li et al., 2006; HernandezCamacho et al., 2017).

\section{Model Evaluation}

Model evaluation occurred in four steps. First, the following fit criteria were used (Brown, 2015): chi-square $\left(\chi^{2}\right)$, comparative fit index (CFI $\geq 0.90)$, root mean squared error of approximation (RMSEA $\leq 0.05)$, and standardized root mean square residual (SRMR < 0.08). We apply these guidelines with caution, as ML-based fit indices have mixed support with robust estimators (Nye and Drasgow, 2011; Zhao, 2015; Xia and Yang, 2019; Shi et al., 2020). Second, the examination of parameters and standard errors provided information about the models' performance, identified areas of misfit, and convergence issues. Third, the $\Delta \chi^{2}$ was employed to compare the conventional BM and restricted $\mathrm{BM}$, given these models were nested (Hernandez-Camacho et al., 2017). A significant $\Delta \chi^{2}(p<0.01)$ would indicate that the restricted BM fit worse compared to the conventional BM. Fourth, we examined the interpretability of the models in relation to theory. After identifying the best fitting model, internal consistency reliability was estimated via omega $(\omega)$ (McDonald, 1999). If the best model was one of the bifactor models, we also estimated omega hierarchical $\left(\omega_{\mathrm{H}}\right)$ to evaluate the variance attribute to the general factor (Rodriguez et al., 2016). Moreover, 
TABLE 3 | Summary of the presence LID for the MDA.

\begin{tabular}{llllll}
\hline & \multicolumn{2}{c}{ Yen's Q3 } & & \multicolumn{2}{c}{ Restricted bifactor } \\
\cline { 2 - 3 } \cline { 5 - 6 } Prompt & $\boldsymbol{N}$ & $\boldsymbol{M}$ & & $\boldsymbol{\sigma}_{\boldsymbol{\gamma}}{ }^{2}$ & Magnitude of LID \\
\hline 1 & 3 & 0.30 & & 4.08 & High \\
2 & 2 & 0.20 & & 3.96 & High \\
3 & 0 & -0.04 & & 0.20 & Low \\
4 & 0 & -0.01 & 0.21 & Low
\end{tabular}

$\sigma_{\gamma}{ }^{2}=$ variance for specific factors. $N=$ number of pairs identified with LID for each prompt. $M=$ averages for $Q 3$ values for each prompt.

we tested additional models if modifications (i.e., reducing the number of specific factors) were justified with the conventional $\mathrm{BM}$ and restricted BM. These additional models were examined in an exploratory framework, as we did not have theory to specify the form of these additional models.

\section{RESULTS}

\section{Test for Local Item Dependence}

Table 3 provides the estimated testlet effect variances and values for the restricted $B M$, and $Q 3$, respectively. The testlet effect variance ranged from 0.21 to 4.08 . The testlet effect variance for the restricted BM identified low LID for prompt 3 and 4, and high LID for prompt 1 and 2. The Q3 averages ranged from -0.03 to 0.30 for items within the prompts. Further inspection of the Q3 values identified three and two pairs with LID for prompt 1 and 2, respectively, and in agreement with the restricted $\mathrm{BM}$ results. Given agreement across methods, the MDA prompts 1 and 2 appear to violate the LII assumption. The one factor should not be considered for use, but its results are presented in tables and briefly mentioned in the next section for comparison and completeness purposes. Based on these findings, the MDA may need a scoring model that accounts for the testlet effect in prompt 1 and prompt 2 .

The multidimensional models were investigated in subsequent analyses to determine the best scoring model for the MDA. The results of RQ1 also guided the modification of the restricted BM and conventional BM, resulting in the reduced versions for these original models. Given that the magnitude of LID was small, reduced versions eliminated the secondary specific factor for prompt three and four.

\section{Modeling the Testlet Effect}

Table 4 summarizes the fit for the one-factor, two original models (i.e., the conventional bifactor with four specific factors and the restricted bifactor with four specific factors) and two reduced models (i.e., conventional bifactor with two specific factors and the restricted bifactor with two specific factors). The original conventional $\mathrm{BM}$ failed to converge, resulting in an ill-defined solution. The one-factor model did not meet fit criteria. Model fit was not excellent for the reduced conventional $\mathrm{BM}$, original restricted $\mathrm{BM}$, and reduced restricted $\mathrm{BM}$ (i.e., all $\chi^{2}$ were significant, $p<0.05, \mathrm{CFI}_{\text {range }}=0.928-0.941$; $\left.\mathrm{RMSEA}_{\text {range }}=0.100-0.113, \mathrm{SRMR}_{\text {range }}=0.117-0.121\right)$. That is, all fit indices did not meet set fit criteria. We note that even though these fit indices did not meet all fit guidelines, we did not continue to modify these specific models because we had no additional theoretical justification for modifications. Instead, we took an exploratory approach.

We conducted a post hoc exploratory factor analysis (EFA). EFA has been used successfully to detect LID where item content and item location have been associated with LID (Stucky et al., 2011). Based on the scree plot and parallel analysis results, two factors were suggested. Inspection of the factor loadings suggested the first 6 indicators (prompt 1 and 2) and the last 6 indicators (prompt 3 and 4) formed factor 1 and factor 2, respectively. These factors had a low correlation $(r=0.36)$. These results were interesting, as they align with our results via the Q3 and restricted bifactor model, suggesting that there is the presence LID for responses within the prompts, and prompt 1 and 2 have a stronger testlet effect compared to Prompt 3 and 4. Thus, the combination of the prompts into two factors could be indictive of locally dependent units representing a testlet or method effect, as seen in previous use of EFA for detecting LID (Stucky et al., 2011).

Given the above result, two additional post hoc CFA models were estimated with the same data. Both models allowed the items to be related to the general factor. First, a restricted bifactor model with two specific factors was specified using indicators from prompt 1 and 2 to form the first specific factor and indicators from prompt 3 and 4 to form the second specific factor. This model failed to converge. Second, a restricted bifactor model with one specific factor was specified using indicators from prompt 1 and 2 to form a specific factor. The fit of the latter model, the combined restricted reduced bifactor model (CRRBM), as seen in Table 4, met all fit criteria. Thus, the CRRBM was retained as the best fitting model for the MDA scores given it (a) was a

TABLE 4 | Model fit indices for the models.

\begin{tabular}{|c|c|c|c|c|c|c|c|}
\hline Model & $x^{2}$ & $d f$ & $P$ & CFI & SRMR & RMSEA & RMSEA-Cl \\
\hline One-factor & 418.52 & 54 & $<0.001$ & 0.835 & 0.180 & 0.162 & $0.148-0.177$ \\
\hline Reduced conventional BM & 205.69 & 48 & $<0.001$ & 0.928 & 0.118 & 0.113 & $0.097-0.129$ \\
\hline Restricted BM & 179.43 & 50 & $<0.001$ & 0.941 & 0.117 & 0.100 & $0.085-0.116$ \\
\hline Reduced restricted BM (RRBM) & 199.02 & 52 & $<0.001$ & 0.933 & 0.121 & 0.105 & $0.090-0.121$ \\
\hline Combined RRBM & 89.79 & 53 & $<0.002$ & 0.983 & 0.073 & 0.052 & $0.033-0.070$ \\
\hline
\end{tabular}

$\mathrm{CFI}=$ the comparative fit index. RMSEA = root mean squared error of approximation. SRMR = standardized root mean square residual. $\mathrm{Cl}=\mathrm{Confidence} \mathrm{interval}$. 
parsimonious model in comparison to the other BM models, (b) accounted for a large presence of LID in prompt 1 and 2, and (c) was the only model to meet fit criteria. We did not engage in model comparisons via chi-square difference tests since the other models did not meet fit criteria and were not acceptable fitting model candidates.

Table 5 presents the standardized loadings for CRRBM, where loadings ranged from 0.19 to 0.92 . The estimated omega $(\omega)$ was 0.93 for the total MDA score. Notice that this estimate, controlling for the method or testlet effect, was similar as the other bifactor models, as seen in Table 5. The $\omega_{\mathrm{H}}$ was 0.69 for the scores from the CRRBM, accounting for the specific factor. Most of the reliable variance (0.74) in the total scores can be attributable to the general MDA factor, $24 \%$ is attributable to the testlet factor, and $7 \%$ is estimated to be random error. We also correlated factor scores of the general factor from the 5 tested models, which had a range of 0.96 to 0.99 . That is, all models resulted in the same factor scores for the general factor. We recognize that more work is needed to continue to explore the underlying structure of the MDA, given our post hoc model fitting.

\section{DISCUSSION}

The purposes of this study were to evaluate the LII assumption and support a scoring inference for the MDA scores for use with university students through accounting for the testlet or method effect with a bifactor model. We focused on the MDA because it (a) is an innovative assessment, (b) contains responses that may violate the LII assumption, and (c) lacks evidence that examines this assumption and information to support a scoring inference. We also demonstrate how to apply a restricted $\mathrm{BM}$ to a non-achievement measure and the modeling of the testlet effect with the BM models. Collectively, our results demonstrate that the MDA's prompt 1 and prompt 2 have the presence of LID, and the combined reduced restricted bifactor model supports a scoring inference for the MDA.

Results supported the presence of LID for the MDA through the restricted $\mathrm{BM}$ and the Q3. The magnitude of LID is high for the MDA's prompt 1 and prompt 2 via the restricted BM. Complementing the restricted BM, Q3 confirmed that LID exits for prompt 1 and prompt 2. The existence of LID in the MDA is aligned with the literature of free response format assessments (Ferrara et al., 1997, 1999). Our results suggest that LID appears most often in prompt 1 and 2 that ask participants to think and provide an explanation about an event where they feel success or failure and required self-evaluation about their abilities (self-concept). In Table 2, we also saw that more students displayed attributes in prompt 1 and 2 compared to prompt 3 and 4. It is possible that this higher rate of occurrence of these attributes, in these prompts is increasing the detection of the testlet effect in these prompts compared to prompts 3 and 4 . A reviewer suggested that this could be a result of rater variance. However, G-theory suggests that variance associated with rater is low in this sample.

Results support a scoring claim demonstrating how the combined reduced restricted $\mathrm{BM}$ can account for the testlet effect. That is, to create factor scores for the MDA, one could use the CRRBM model, compared to the unidimensional model. A model that controls for the testlet or a method effect may produce better factor score estimates. That said, the testlet effect, at least in this MDA dataset, may not be strong enough to warrant the more complicated scoring model, as seen with the high correlations the factor scores (e.g., $>0.97$ ) between the unidimensional model and the bifactor alternative models. The original restricted bifactor was not considered the best model

TABLE $\mathbf{5}$ | Standardized factor loadings from the confirmatory factor analyses.

\begin{tabular}{|c|c|c|c|c|c|c|c|c|c|c|c|c|c|c|}
\hline \multicolumn{2}{|c|}{ 1-Factor } & \multicolumn{3}{|c|}{ Reduced CBM } & \multicolumn{3}{|c|}{ Reduced RBM } & \multicolumn{5}{|c|}{ RBM } & \multicolumn{2}{|c|}{ Combined RBM } \\
\hline MDA & $\mathbf{F 1}$ & $\mathbf{F} 1$ & SP1 & SP2 & $\mathbf{F 1}$ & SP1 & SP2 & $\mathbf{F 1}$ & SP1 & SP2 & SP3 & SP4 & $\mathbf{F} 1$ & SP12 \\
\hline Item1 & 0.640 & 0.372 & 0.826 & & 0.373 & 0.822 & & 0.401 & 0.810 & & & & 0.306 & 0.834 \\
\hline Item2 & 0.518 & 0.284 & 0.736 & & 0.315 & 0.694 & & 0.337 & 0.682 & & & & 0.253 & 0.689 \\
\hline Item3 & 0.623 & 0.383 & 0.744 & & 0.356 & 0.783 & & 0.382 & 0.771 & & & & 0.287 & 0.782 \\
\hline Item4 & 0.292 & 0.162 & & 0.701 & 0.240 & & 0.524 & 0.259 & & 0.516 & & & 0.186 & 0.508 \\
\hline Item5 & 0.425 & 0.355 & & 0.461 & 0.297 & & 0.648 & 0.323 & & 0.642 & & & 0.213 & 0.580 \\
\hline Item6 & 0.402 & 0.322 & & 0.763 & 0.329 & & 0.720 & 0.352 & & 0.700 & & & 0.203 & 0.554 \\
\hline Item7 & 0.759 & 0.787 & & & 0.788 & & & 0.750 & & & 0.335 & & 0.796 & \\
\hline Item8 & 0.902 & 0.916 & & & 0.916 & & & 0.861 & & & 0.384 & & 0.919 & \\
\hline Item9 & 0.903 & 0.920 & & & 0.921 & & & 0.865 & & & 0.386 & & 0.925 & \\
\hline Item10 & 0.795 & 0.821 & & & 0.821 & & & 0.790 & & & & 0.363 & 0.826 & \\
\hline Item11 & 0.810 & 0.838 & & & 0.837 & & & 0.792 & & & & 0.364 & 0.844 & \\
\hline Item12 & 0.869 & 0.890 & & & 0.890 & & & 0.842 & & & & 0.387 & 0.895 & \\
\hline$\omega$ & 0.909 & 0.938 & & & 0.937 & & & 0.943 & & & & & 0.931 & \\
\hline$\omega_{\mathrm{H}}$ & & 0.794 & & & 0.797 & & & 0.768 & & & & & 0.688 & \\
\hline
\end{tabular}

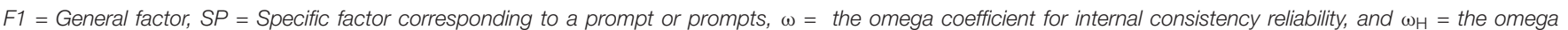
hierarchical coefficient for internal consistency reliability.

$C B M=$ Conventional Bifactor; $R B M=$ Restricted Bifactor. 
for the MDA, as the presence of LID in two of the prompts was weak, and not likely to bias the parameters estimates (e.g., Koziol, 2016). The CRRBM allows all items to be related to one general construct and accounts for the testlet effect in prompt 1 and 2, where this effect is the strongest, by modeling this as one specific factor. Of course, this best fitting model was the result of exploratory and post hoc model specification and testing. This model would need to be replicated in a new and independent sample.

\section{Implications}

Three primary implications for researchers and practitioners were identified. First, following the recommendations in the method effect literature (Podsakoff et al., 2003), we encourage future work to consider careful revisions for prompt 1 and 2 for the MDA to minimize the impact of the testlet effect. However, we acknowledged that revisions must be guided by the intended use of the MDA and underlying theory. It may be worth exploring the differences between prompts 1 and 2 and prompts 3 and 4 through techniques such as cognitive interviews, given the latter prompts assess overlapping constructs, but show small amount of LID and a lower attribute prevalence rate.

Second, we advocate the use of multiple statistics (i.e., restricted $\mathrm{BM}$ and other LID statistics) to detect LID for testlet data. Using the restricted BM and other LID statistics builds on the strengths of each approach to detect for LID. The LID statistic like Q3 helps to detect specific pairs of items with LID without information about the factor structure of the MDA. However, the restricted $\mathrm{BM}$ provides the magnitude of LID for a testlet without detailed information about the item pairs, but it can help examine and support a factor structure for the MDA. The use of both approaches can provide additional information about the degree of LID and help ensure accurate detection of LID. This combination approach is like the use of multiple indices to judge model fit in SEM or the use of multiple criteria in evaluating factor models for invariance.

Third, we encouraged scholars and practitioners to incorporate the conventional $\mathrm{BM}$ and restricted $\mathrm{BM}$ into their research tool kit and apply these models as a control for method effects, such as the testlet effect, when appropriate. Such practice is an improvement over ignoring the testlet effect in a scoring model. Modeling the testlet effect can lead to accurate parameters and reliability estimates (e.g., Hernandez-Camacho et al., 2017). In fact, we saw that the omega estimates for the MDA scores when the method or testlet effect was controlled for was higher compared to the unidimensional factor model (e.g., 0.90 vs. 0.93). We acknowledge that estimation of models requires adequate sample sizes. Although the conventional BM can model the testlet effect, incorporating the additional parameters (e.g., two loadings for each item) requires a reasonable number of individuals to avoid convergence issues and produce accurate estimates (De la Torre and Hong, 2010; Jung et al., 2020). Thus, the sample size constraints would make the conventional BM approach less desirable to assess testlet data, compared to other approaches at this time. Given this, the restricted BM may assist with modeling testlet data. This may be helpful in applications where sample sizes are small, especially in non-achievement assessment programs (e.g., Mini-Mental State Examination; Rubright et al., 2016) and the MDA.

\section{Limitations}

We identify several limitations of this study. First, the participants may not represent students from the diverse type of universities and regions in the U.S, given they were sampled from a single university located in a Western United States state. Other university students might have different experiences given contextual and cultural factors that may play a critical role in the degree to which MD attributes manifests within students, and hence the testlet effect could be different. In future studies, researchers should examine the scoring inference of the MDA among samples of students from other universities.

Second, measurement invariance of the MDA across groups was not considered. Such information informs if the factor structure, including the testlet effect of the MDA performs the same across different populations. Besides invariance, responses styles (e.g., Baird et al., 2017) could be an issue, as styles may also differ across student populations. In the absence of such evidence, the MDA scores may lack some precision, which requires additional work to understand. Future studies can investigate if the reduced restricted $\mathrm{BM}$ functions the same between groups, such as gender and ethnic groups. It may be the case that some interpretations of the prompts by students with certain backgrounds could yield less (or more) presence of a testlet effect for the MDA.

Third, our sample size was not large, and was based on a convenience sampling framework at a single public university. The sample size may have led to the convergence issues for the conventional BM with four specific factors, given large sample sizes are often needed to estimate the multidimensional models. The generalizability of our findings could be improved with a more diverse population of students drawn from more than one university.

Fourth, we used the standard cut-off criteria to identify LID via the restricted bifactor model and the Q3 statistic in absence of other guidelines and strict thresholds for the Q3 (e.g., Christensen et al., 2017). We acknowledge that the criteria (a) for the restricted bifactor model lack strong empirical support, and (b) for the Q3 does not have specific thresholds. Given this, we opted to use the criteria defined by the Bradlow' study for the restricted bifactor model (Bradlow et al., 1999) and commonly used thresholds in practice for Q3. Additional methodological work may help to understand how criteria function for these approaches under different conditions and with different types of assessments to accurately identify LID for testlet data.

Fifth, our modeling modification process was conducted in an exploratory framework. We did not have theory to guide additional model specifications. Instead, we used a two-step approach with exploratory factor analysis and CFA to identify a model that met fit criteria. However, given the novel application to account for the data structure we anticipated possible modifications would be suggested. Given the ex post facto modifications, we recognize that generalizability of CRRBM is questionable without validation or replication with independent samples. Our EFA results suggested that unidimensionality may 
not be supported, yet the identified two factors where indictive of testlet or methods effects as seen in previous work (Stucky et al., 2011). This was supported by the CRRBM, which fit well. Future work with the MDA will need to continue to explore the structure of the MDA.

\section{CONCLUSION}

The MDA may assist in collecting a more in-depth understanding of students' MD attributes compared to self-report questionnaires and performance tasks (Duckworth and Yeager, 2015). That said, the nature of such data might limit the usage of traditional scoring models, such as the unidimensional model, given the LII assumption can be violated. In this situation, the testlet effect, a type of method effect, can be present for the MDA's writing prompts and must be controlled in a scoring model. In the CFA framework, other models (e.g., multi-trait multi-method) have been proposed to account for method effect where it is modeled as a specific factor (Podsakoff et al., 2003; Maul, 2013). Similar to such work, both conventional and restricted BM can control the testlet effect with specific factors, as we have demonstrated.

Both the conventional and restricted BM are alternative models that can be used with achievement and non-achievement measures. Our study demonstrates how to apply these models to non-achievement measures, as with the MDA. Beyond demonstrating how to control for this method effect with bifactor models, we provide valuable insight about the presence of LID within prompts and factor structure for the MDA, and likely other such measures. Given the call for more innovative measures beyond rating scales, this may be useful for other areas of assessment. Given the existence of LID, the CRRBM can be useful

\section{REFERENCES}

Baars, M. A. E., Nije Bijvank, M., Tonnaer, G. H., and Jolles, J. (2015). Self-report measures of executive functioning are a determinant of academic performance in first-year students at a university of applied sciences. Front. Psychol. 6:1131. doi: 10.3389/fpsyg.2015.01131

Baird, B. M., Lucas, R. E., and Donnellan, M. B. (2017). The role of response styles in the assessment of intraindividual personality variability. J. Res. Pers. 69, 170-179. doi: 10.1016/j.jrp.2016.06.015

Baldonado, A. A., Svetina, D., and Gorin, J. (2015). Using necessary information to identify item dependence in passage-based reading comprehension tests. Appl. Measur. Educ. 28, 202-218. doi: 10.1080/08957347.2015.1042154

Bandalos, D. L., and Finney, S. J. (2001). "Item parceling issues in structural equation modeling," in Advanced Structural Equation Modeling: New Developments and Techniques, eds G. A. Marcoulides and R. E. Schumacker (Mahwah, NJ: Lawrence Erlbaum Associates, Inc), 269-296.

Bradlow, E. T., Wainer, H., and Wang, X. (1999). A Bayesian random effects model for testlets. Psychometrika 64, 153-168. doi: 10.1007/bf02294533

Braeken, J., Tuerlinckx, F., and Boeck, P. (2007). Copula functions for residual dependency. Psychometrika 72, 393-411. doi: 10.1007/s11336-007-9005-4

Brown, T. A. (2015). Confirmatory Factor Analysis for Applied Research, 2nd Edn. New York, NY: Guilford Publications.

Christensen, K. B., Makransky, G., and Horton, M. (2017). Critical values for Yen's $Q_{3}$ : identification of local dependence in the Rasch model using residual correlations. Appl. Psychol. Meas. 41, 178-194. doi: 10.1177/0146621616677520

Cromley, J. G., Perez, T., and Kaplan, A. (2015). Undergraduate STEM achievement and retention. Policy Insight. Behav. Brain Sci. 3, 4-11. doi: 10. $1177 / 2372732215622648$ to provide accurate parameter estimates for the MDA. Additional validity evidence is needed to support the MDA score use among other university students and ethnic groups.

\section{DATA AVAILABILITY STATEMENT}

The original contributions presented in the study are included in the article/supplementary material, further inquiries can be directed to the corresponding author/s.

\section{ETHICS STATEMENT}

The studies involving human participants were reviewed and approved by the Washington State University Institutional Review Board. The patients/participants provided their written informed consent to participate in this study.

\section{AUTHOR CONTRIBUTIONS}

DA was responsible for the main components of the writing and analysis. BF was responsible for assisting with the conceptualization of the idea and writing. Both authors contributed to the article and approved the submitted version.

\section{FUNDING}

There was no direct funding for this work. It was a component of DA's dissertation.

Crossley, J. (2016). Assessing the non-cognitive domains: measuring what matters well. Innov. Pract. Higher Educ. Asses. Measur. 16, 348-372. doi: 10.4018/9781-5225-0531-0.ch018

Dai, S., Svetina, D., and Wang, X. (2019). Reporting subscores using R: a software review. J. Educ. Behav. Statist. 42, 617-638. doi: 10.3102/1076998617716462

de Ayala, R. (2009). The Theory and Practice of Item Response Theory (Methodology in the Social Sciences). New York, NY: Guilford Press.

De la Torre, J., and Hong, Y. (2010). Parameter estimation with small sample size a higher-order irt model approach. Appl. Psychol. Measur. 34, 267-285. doi: 10.1177/0146621608329501

DeMars, C. E. (2006). Application of the bi-factor multidimensional item response theory model to testlet-based tests. J. Educ. Measur. 43, 145-168.

Duckworth, A. L., and Yeager, D. S. (2015). Measurement matters. Educ. Res. 44, 237-251. doi: 10.3102/0013189x15584327

Eckes, T. (2011). Item banking for C-tests: a polytomous rasch modeling approach. Psychol. Sci. 53:414.

Farrington, C. A., Roderick, M., Allensworth, E., Nagaoka, J., Keyes, T. S., Johnson, D., et al. (2012). Teaching Adolescents to Become Learners: The Role of Noncognitive Factors in Shaping School Performance. Chicago: University of Chicago Consortium on Chicago School Research.

Fernandez-Berrocal, P., and Extremera, N. (2006). Emotional intelligence: a theoretical and empirical review of its first 15 years of history. Psicothema 18, $7-12$.

Ferrara, S., Huynh, H., and Baghi, H. (1997). Contextual characteristics of locally dependent open-ended item clusters in a large-scale performance. Appl. Measur. Educ. 10, 123-144. doi: 10.1207/s15324818ame1002_2

Ferrara, S., Huynh, H., and Michaels, H. (1999). Contextual explanations of local dependence in item clusters in a large scale hands-on science performance 
assessment. J. Educ. Measur. 36, 119-140. doi: 10.1111/j.1745-3984.1999. tb00550.x

García, E. (2016). The need to address non-cognitive skills in the education policy agenda. Non-Cogn. Skills Fact. Educ. Attain. 16, 31-64. doi: 10.1007/978-946300-591-3_3

Gibbons, R. D., and Hedeker, D. R. (1992). Full-information item bi-factor analysis. Psychometrika 57, 423-436. doi: 10.1007/bf02295430

Glas, C. A. W., Wainer, H., and Bradlow, E. T. (2000). "MML and EAP estimation in testlet-based adaptive testing," in Computerized Adaptive Testing: Theory and Practice, eds W. J. van der Linden and C. A. W. Glas (Alphen aan den Rijn: Kluwer), 271-287.

Hall, R. J., Snell, A. F., and Foust, M. S. (1999). Item parceling strategies in SEM: investigating the subtle effects of unmodeled secondary constructs. Organ. Res. Methods 2, 233-256. doi: 10.1177/109442819923002

Hernandez-Camacho, A., Olea, J., and Abad, F. J. (2017). Comparison of uniand multidimensional models applied in testlet-based tests. Methodology 13, 135-143. doi: 10.1027/1614-2241/a000137

Jung, S., Seo, D. G., and Park, J. (2020). Regularized exploratory bifactor analysis with small sample sizes. Front. Psychol. 11:507. doi: 10.3389/fpsyg.2020.00507

Kane, M. T. (2013). Validating the interpretations and uses of test scores. J. Educ. Measur. 50, 1-73. doi: 10.1111/jedm.12000

Kaplan, A., and Patrick, H. (2016). "Learning environments and motivation," in Handbook of Motivation at School, 2nd Edn, eds K. Wentzel and D. Miele (Abingdon: Routlege), 251-274.

Koziol, N. A. (2016). Parameter recovery and classification accuracy under conditions of testlet dependency: a comparison of the traditional 2PL, testlet, and bi-factor models. Appl. Measur. Educ. 29, 184-195. doi: 10.1080/08957347. 2016.1171767

Li, Y., Bolt, D. M., and Fu, J. (2006). A comparison of alternative models for testlets. Appl. Psychol. Measur. 30, 3-21. doi: 10.1177/0146621605275414

Lord, F., and Novick, M. (1968). Statistical Theories of Mental Test Scores. Boston, MA: Addison-Wesley.

Luo, Y. (2018). A short note on estimating the testlet model with different estimators in Mplus. Educ. Psychol. Measur. 78, 517-529. doi: 10.1177/ 0013164417717314

Luo, Y., and Gordon-Wolf, M. (2019). Item parameter recovery for the two parameter testlet model with different estimation methods. Psychol. Sci. 61, 65-89.

Maul, A. (2013). Method effects and the meaning of measurement. Front. Psychol. 4:169. doi: 10.3389/fpsyg.2013.00169

McDonald, R. P. (1999). Test Theory: A Unified Treatment. Mahwah, NJ: Erlbaum.

Muthén, B., and Asparouhov, T. (2002). Latent Variable Analysis With Categorical Outcomes: Multiple-Group and Growth Modeling in Mplus. Unpublished manuscript. Los Angeles.

Muthén, L. K., and Muthén, B. O. (2017). Mplus: Statistical Analysis With Latent Variables: User's Guide (Version 8). Los Angeles, CA: Authors.

National Academies of Sciences, Engineering, and Medicine (2017). Supporting Student's College Success: The Role of Assessment of Interpersonal and Interpersonal Competencies. Washington, DC: The National Academies Press. doi: $10.17226 / 24697$

Nunnally, J. C., and Bernstein, I. H. (1994). Psychometric Theory, 3rd Edn. New York, NY: McGraw-Hill.

Nye, C. D., and Drasgow, F. (2011). Assessing goodness of fit: simple rules of thumb simply do not work. Organiz. Res. Methods 14, 548-570.

Podsakoff, P. M., Mackenzie, S. B., and Podsakoff, N. P. (2012). Sources of method bias in social science research and recommendations on how to control it. Annu. Rev. Psychol. 63, 539-569. doi: 10.1146/annurev-psych-120710-100452

Podsakoff, P. M., MacKenzie, S. B., Lee, J., and Podsakoff, N. P. (2003). Common method biases in behavioral research. J. Appl. Psychol. 88, 879-903. doi: 10.1037/ 0021-9010.88.5.879
Raykov, T., Marcoulides, G. A., and Patelis, T. (2015). The importance of the assumption of uncorrelated errors in psychometric theory. Educ. Psychol. Measur. 75, 634-647. doi: 10.1177/0013164414548217

Rijmen, F. (2010). Formal relations and an empirical comparison among the bifactor, the testlet, and a second-order multidimensional IRT model. J. Educ. Measur. 47, 361-372. doi: 10.1111/j.1745-3984.2010.00118.x

Rodriguez, A., Reise, S. P., and Haviland, M. G. (2016). Evaluating bifactor models: calculating and interpreting statistical indices. Psychol. Methods 21, 137-150. doi: $10.1037 /$ met0000045

Rosenbaum, P. R. (1988). Items bundles. Psychometrika 53, 349-359.

Rubright, J. D., Nandakumar, R., and Karlawish, J. (2016). Identifying an appropriate measurement modeling approach for the mini-mental state examination. Psychol. Asses. 28, 125-133. doi: 10.1037/pas0000146

Schroeders, U., Robitzsch, A., and Schipolowski, S. (2014). A comparison of different psychometric approaches to modeling testlet structures: an example with c-tests. J. Educ. Measur. 51, 400-418. doi: 10.1111/jedm.12054

Shi, D., Maydeu-Olivares, A., and Rosseel, Y. (2020). Assessing fit in ordinal factor analysis models: SRMR vs. RMSEA. Struct. Equat. Model. Multidis. J. 27, $1-15$.

Stucky, B. D., Gottfredson, N. C., Panter, A. T., Daye, C. E., Allen, W. R., and Wightman, L. F. (2011). An item factor analysis and item response theory-based revision of the everyday discrimination scale. Cult. Diver. Ethnic Minor. Psychol. 17, 175-185. doi: 10.1037/a0023356

Wainer, H., and Kiely, G. L. (1987). Item clusters and computerized adaptive testing: a case for testlets. J. Educ. Measur. 24, 185-201. doi: 10.1111/j.17453984.1987.tb00274.x

Wainer, H., and Wang, X. (2000). Using a new statistical model for testlets to score toefl. J. Educ. Measur. 37, 203-220. doi: 10.1111/j.1745-3984.2000.tb01083.x

Xia, Y., and Yang, Y. (2019). RMSEA, CFI, and TLI in structural equation modeling with ordered categorical data: the story they tell depends on the estimation methods. Behav. Res. Methods 51, 409-428.

Yen, W. M. (1984). Effects of local item dependence on the fit and equating performance of the three-parameter logistic model. Appl. Psychol. Measur. 8, $125-145$.

Yen, W. M. (1993). Scaling performance assessments: strategies for managing local item dependence. J. Educ. Measur. 30, 187-213. doi: 10.1111/j.1745-3984.1993. tb00423.x

Zhao, Y. (2015). The Performance of Model Fit Measures by Robust Weighted Least Squares Estimators in Confirmatory Factor Analysis. University Park, PA: The Pennsylvania State University.

Conflict of Interest: The authors declare that the research was conducted in the absence of any commercial or financial relationships that could be construed as a potential conflict of interest.

The handling editor declared a past co-authorship with one of the authors BF.

Publisher's Note: All claims expressed in this article are solely those of the authors and do not necessarily represent those of their affiliated organizations, or those of the publisher, the editors and the reviewers. Any product that may be evaluated in this article, or claim that may be made by its manufacturer, is not guaranteed or endorsed by the publisher.

Copyright (C) 2021 Alpizar and French. This is an open-access article distributed under the terms of the Creative Commons Attribution License (CC BY). The use, distribution or reproduction in other forums is permitted, provided the original author(s) and the copyright owner(s) are credited and that the original publication in this journal is cited, in accordance with accepted academic practice. No use, distribution or reproduction is permitted which does not comply with these terms. 